\title{
Anesthesia Preoperative Clinic Referral for Elevated Hbaic Reduces Complication Rate in Diabetic Patients Undergoing Total Joint Arthroplasty
}

\author{
Peter J. Kallio ${ }^{1,3}$; Jenea Nolan ${ }^{2}$; Amy C. Olsen ${ }^{2}$; Susan Breakwell ${ }^{3}$; Richard Topp ${ }^{3}$; Paul S. \\ Pagel ${ }^{1, *}$ \\ ${ }^{1}$ Anesthesia Service, Clement J. Zablocki Veterans Affairs Medical Center, Milwaukee, Wisconsin, USA \\ ${ }^{2}$ The School of Nurse Anesthesia, Rosalind Franklin University is located in Chicago, Illinois, USA \\ ${ }^{3}$ Department of Nursing, Marquette University, Wisconsin, USA \\ ${ }^{*}$ Corresponding author: Paul S. Pagel, Anesthesia Service, Clement J. Zablocki Veterans Affairs Medical Center, Milwaukee, Wisconsin, USA. Tel: +1-4143842000, Fax: +1-4143842939, \\ E-mail: pspagel@mcw.edu
}

Received: October 7, 2014; Revised: November 11, 2014; Accepted: December 19, 2014

\begin{abstract}
Background: Diabetes mellitus (DM) is risk factor for complications after orthopedic surgery.
Objectives: We tested the hypothesis that anesthesia preoperative clinic (APC) referral for elevated glycosylated hemoglobin (HbA1c) reduces complication rate after total joint arthroplasty (TJA).

Patients and Methods: Patients $(n=203)$ with and without DM were chosen from 1,237 patients undergoing TJA during 2006-12. Patients evaluated in the APC had surgery in 2006-8 regardless of HbA1c (uncontrolled). Those evaluated between in subsequent two-year intervals were referred to primary care for HbA1c $\geq 10 \%$ and $\geq 8 \%$, respectively, to improve DM control before surgery. Complications and mortality were quantified postoperatively and at three, six, and twelve months. Length of stay (LOS) and patients requiring a prolonged LOS (>5 days) were recorded.

Results: Patients (197 men, 6 women) underwent 71,131, and 1 total hip, knee, and shoulder replacements, respectively. Patients undergoing TJA with uncontrolled HbAic and those with $\mathrm{HbA1c}<10 \%$, but not those with $\mathrm{HbA1c}<8 \%$, had a higher incidence of coronary disease and hypercholesterolemia than patients without DM. An increase in complication rate was observed in DM patients with uncontrolled HbA1c versus patients without DM $(\mathrm{P}<0.001)$; the complication rate progressively decreased with tighter HbAic control. More DM patients with preoperative HbAic that was uncontrolled or $\geq 10 \%$ required prolonged LOS versus those without $\mathrm{DM}(\mathrm{P}<0.001$ and $\mathrm{P}=0.0404$, respectively).

Conclusions: APC referral for elevated HbA1c reduces complication rate and the incidence of prolonged hospitalization during the first year after surgery in diabetics undergoing TJA.
\end{abstract}

Keywords: Diabetes Mellitus; Glycosylated Hemoglobin; Orthopedic Surgery; Arthroplasty

\section{Background}

Diabetes mellitus is an important risk factor for postoperative complications after orthopedic surgery (1-5). Patients with diabetes undergoing primary or revision total joint arthroplasty had higher hospital costs, fewer routine discharges, and an increased risk of stroke, pneumonia, ileus, urinary tract infection, and transfusion than those without the disease $(1,5)$. Uncontrolled diabetes [as determined using patient self-reporting of blood glucose concentration, glycosylated hemoglobin (HbA1c) level, and end-organ damage] was shown to be particularly harmful, as the incidence of morbidity and mortality was greater in diabetic patients undergoing arthroplasty with poorly- versus well-controlled disease (4). Recent evidence further suggests that poor long-term control of diabetes may increase postoperative complications in orthopedic and other types of surgery $(2,3)$. A preoperative HbA1c $\geq 7.1 \%$ was implicated as a major risk factor for all-cause complications in diabetic patients undergoing total joint arthroplasty (2), whereas a HbA1c level $\leq 7.0 \%$ may decrease the risk of perioperative and long-term complications in patients undergoing cardiac and noncardiac surgery (6-9). Anesthesia-directed preoperative clinics evaluation enhance operating room efficiency, improve patient education and satisfaction, reduce unnecessary testing and consultation, and decrease adverse perioperative events (10-13) in patients undergoing elective surgery, including those scheduled for orthopedic procedures (14). Whether the anesthesia preoperative evaluation clinic and referral to primary care are capable of reducing the adverse effects of diabetes by improving chronic control of blood glucose concentration is unknown.

\section{Objectives}

The authors tested the hypothesis that preoperative

Copyright (C) 2015, Iranian Society of Regional Anesthesia and Pain Medicine(ISRAPM). This is an open-access article distributed under the terms of the Creative Commons Attribution-NonCommercial 4.0 International License (http://creativecommons.org/licenses/by-nc/4.0/) which permits copy and redistribute the material just in noncommercial usages, provided the original work is properly cited. 
Kallio PJ et al.

evaluation clinic screening and referral of veterans with poorly controlled diabetes to their primary care physicians to obtain better long-term control of the disease before surgery reduces complication rate during the first year after elective total joint arthroplasty.

\section{Patients and Methods}

The Institutional Review Boards of Clement J. Zablocki Veterans Affairs Medical Center and Marquette University reviewed and approved the protocol. Written informed consent was waived because of the retrospective, observational study design. The study protocol conforms to the ethical guidelines of the 1975 Declaration of Helsinki. The electronic medical records of all patients undergoing total joint arthroplasty $(n=1,237)$ at the Zablocki Veterans Affairs Medical Center between January 1, 2006 and December 31, 2012 were identified using an orthopedic surgery database. Two hundred and three patients with and without diabetes ( $n=103$ and $n=100$, respectively) undergoing elective joint arthroplasty were randomly chosen from this cohort. Patients undergoing revision arthroplasty and those with preexisting wound infections were excluded from analysis. All demographic and clinical outcome data for each patient were obtained from simultaneous review of the electronic medical record by three investigators (PJK, JN, AO) to assure consistency. Patients with diabetes were required to have a HbAic level recorded in the medical record within six months before surgery. In January, 2009, based on emerging data about the possible importance of long-term control of blood glucose concentration on outcomes after surgery $(4,7$, 8 ), the preoperative evaluation clinic director (PJK) began to recommend to his patients that those with HbA1c $\geq 10$ should delay their procedures. These patients were counseled to return to their primary care physicians or the endocrinology clinic to obtain better long-term control of blood glucose concentration before proceeding with elective total joint arthroplasty. An analysis of pilot data conducted two years later suggested that this approach for patients with poorly controlled diabetes may be reducing the rate of complications. As a result, the threshold for referral was reduced to HbAlc $\geq 8 \%$ during 2011 and 2012. We chose a threshold value HbA1c $\geq 8 \%$ (rather than the 7\% level recommended by the American Diabetes Association (15)) because aggressive compared with moderate glycemic control may inadvertently cause hypoglycemia and increase perioperative morbidity and mortality in high-risk patients $(16,17)$, such as elderly veterans undergoing total joint arthroplasty. Surgical site or urinary tract infections (verified with appropriate culture and sensitivity), delayed wound healing (including inflammation, dehiscence, and chronic drainage), requirement for transfusion of autologous blood products, unplanned prolonged hospitalization for inadequate pain control, chronic pain requiring long-term management, pneumonia, deep venous thrombosis with or with- out pulmonary embolism, cardiovascular complications including myocardial ischemia or infarction and atrial or ventricular arrhythmias requiring medical treatment (antiarrhythmic medications, cardioversion, electrophysiology intervention, or device placement), cerebrovascular accident, and mortality were quantified during the immediate postoperative period and at the three, six, and twelve month orthopedic surgery follow-up appointments. Hospital length of stay and the number of patients requiring a prolonged length of stay (defined as greater than five days) after surgery were also recorded. Diabetic patients undergoing total joint arthroplasty in the current investigation were treated with either continuous infusions or sliding-scale bolus doses of insulin to maintain blood glucose concentration between 140 to $180 \mathrm{mg} / \mathrm{dL}$ during and after surgery regardless of preoperative HbA1c, $(18,19)$. "Tighter" control of blood glucose concentration was avoided because of the potential risk of hypoglycemia. Consultant internists or endocrinologists were responsible for postoperative diabetic care in these patients and used similar methods and goals for control of blood glucose concentration during hospitalization. Primary care physicians or endocrinologists managed blood glucose concentration using established American Diabetes Association guidelines 15 after the patients were discharged from the hospital.

\subsection{Statistical Analysis}

A power analysis conducted before beginning the study indicated that a group size $\geq 65$ (patients with versus without diabetes) was required for a minimal difference of $20 \%$ in all-cause complication rate between patients with or without diabetes (alpha error $<0.05$; beta error $<0.20$ ) with a power of $95 \%$. Continuous data between groups were analyzed using analysis of variance (ANOVA) followed by Bonferroni's test for differences between means. All continuous data are presented as mean \pm standard deviation. Differences in categorical variables were determined using chi-square analysis. Categorical variables are presented as raw numbers or percentages. Linear regression analysis was used to assess the correlation between the number of complications per patient and HbA1c. Stat Plus: Mac software (Analyst Soft, Alexandria, Virginia, USA) was used for statistical analysis. The null hypothesis was rejected when $\mathrm{P}<0.05$.

\section{Results}

Veterans (197 men and 6 women) underwent 71, 131, and 1 total hip, knee, and shoulder replacements, respectively (Table 1). The demographics in patients without diabetes were similar to those with diabetes irrespective of HbA1c control (Table 1). Serum creatinine was significantly greater in patients with uncontrolled diabetes undergoing elective surgery compared with patients who were not diabetic $(P=0.00121)$. Patients with diabetes with uncontrolled HbA1c and those with HbA1c $<10 \%$, but not 
$<8 \%$, had a higher incidence of coronary artery disease and hypercholesterolemia and were more likely to receive ACE inhibitors or ARB. An increase in complication rate was observed in diabetic patients with uncontrolled HbA1c versus patients without diabetes $(\mathrm{P}<0.0001)$, but this elevated complication rate progressively declined with tighter HbA1c control (Tables 1 and 2). Diabetic patients with preoperative uncontrolled HbA1c or HbA1c <
$10 \%$ also required prolonged length of stay, but the mean length of stay was similar between groups. There were no differences in the incidence of system-specific complications between diabetic patients regardless of HbA1c control compared with patients without diabetes (Table 3). A significant correlation was observed between the number $(\mathrm{n})$ of complications per patient and $\operatorname{HbA1c}(\mathrm{n}=0.339$ $\times$ HbA1c $-1.46 ; \mathrm{r}=0.32, \mathrm{P}<0.01$ ).

Table 1. Demographic, Historical, and Medication data for Patients With and Without Diabetes Based on A1c Control Before Elective total Joint Surgery a, b

\begin{tabular}{|c|c|c|c|c|}
\hline Variables & $-\mathrm{DM}$ & +DM No Referral for A1C & + DM Referral for A1c $<10 \%$ & +DM Referral for A1c $<8 \%$ \\
\hline Number & 100 & 55 & 32 & 16 \\
\hline Surgery Type (Hip, Knee, Shoulder) & $41 / 58 / 1$ & $14 / 41 / 0$ & $11 / 21 / 0$ & $6 / 10 / 0$ \\
\hline \multicolumn{5}{|l|}{ Gender } \\
\hline Male & 99 & 52 & 31 & 15 \\
\hline Female & 1 & 3 & 1 & 1 \\
\hline Age, y & $65 \pm 9$ & $66 \pm 10$ & $66 \pm 8$ & $66 \pm 10$ \\
\hline Weight, kg & $102 \pm 20$ & $103 \pm 21$ & $113 \pm 28$ & $115 \pm 26$ \\
\hline Height, cm & $178 \pm 7$ & $176 \pm 9$ & $177 \pm 9$ & $177 \pm 10$ \\
\hline Body Mass Index, kg/m² & $32 \pm 6$ & $34 \pm 7$ & $36 \pm 7^{c}$ & $36 \pm 7^{c}$ \\
\hline A1c, $\%$ & $5.7 \pm 0.4$ & $7.0 \pm 1.0^{C}$ & $6.9 \pm 0.8^{C}$ & $6.8 \pm 0.6^{c}$ \\
\hline Hematocrit, \% & $42 \pm 4$ & $42 \pm 5$ & $41 \pm 4$ & $41 \pm 4$ \\
\hline Creatinine, mg/dL & $1.04 \pm 0.25$ & $1.24 \pm 0.40^{\mathrm{C}}$ & $1.04 \pm 0.31^{d}$ & $0.88 \pm 0.22^{d}$ \\
\hline Potassium, mEq/L & $4.1 \pm 0.4$ & $4.2 \pm 0.4$ & $4.1 \pm 0.4$ & $4.1 \pm 0.4$ \\
\hline Length of Stay, d & $7.6 \pm 6.4$ & $10.2 \pm 11.4$ & $8.3 \pm 5.7$ & $8.3 \pm 5.9$ \\
\hline Prolonged Length of Stay & 23 & $31^{\mathrm{C}}$ & $14^{\mathrm{C}}$ & 6 \\
\hline Complication Rate & $0.36 \pm 0.63$ & $1.27 \pm 1.18^{\mathrm{C}}$ & $0.78 \pm 1.01$ & $0.50 \pm 0.89$ \\
\hline Coronary Artery Disease & 14 & $14^{\mathrm{C}}$ & $13^{\mathrm{C}}$ & 4 \\
\hline Hypertension & 71 & 45 & 27 & 14 \\
\hline Hypercholesterolemia & 42 & $33^{\mathrm{C}}$ & $23^{c}$ & 9 \\
\hline $\begin{array}{l}\text { Chronic Obstructive Pulmonary } \\
\text { Disease }\end{array}$ & 5 & 6 & 4 & 0 \\
\hline Peripheral Vascular Disease & 4 & 3 & 1 & 1 \\
\hline Tobacco Use & 29 & 16 & 8 & 2 \\
\hline Insulin & 0 & 11 & 7 & 7 \\
\hline Oral Hypoglycemic Drug & 0 & 21 & $23^{d}$ & 10 \\
\hline Beta-Adrenoceptor Antagonist & 34 & 24 & 17 & 6 \\
\hline Calcium Channel Blocker & 23 & 7 & 7 & 3 \\
\hline Nitrate & 1 & 6 & 5 & 2 \\
\hline ACE inhibitor or ATII blocker & 32 & $32^{\mathrm{C}}$ & $22^{C}$ & 8 \\
\hline
\end{tabular}


Kallio PJ et al.

\begin{tabular}{|c|c|c|c|c|c|c|}
\hline Variables & $\begin{array}{c}\text {-DM vs. +DM No } \\
\text { Referral }\end{array}$ & $\begin{array}{c}\text {-DM vs. +DM } \\
\text { Referral for } \\
\text { A1c }<10 \% \\
\end{array}$ & $\begin{array}{c}- \text { DM vs. }+ \text { DM } \\
\text { Referral for } \\
\text { A1c }<8 \% \\
\end{array}$ & $\begin{array}{c}\text { +DM No Referral } \\
\text { vs. +DM Referral } \\
\text { for A1c }<\mathbf{1 0} \% \\
\end{array}$ & $\begin{array}{c}\text { +DM No Referral } \\
\text { vs. +DM Referral } \\
\text { for A1c }<8 \% \\
\end{array}$ & $\begin{array}{c}+ \text { DM Referral for A1c } \\
<\mathbf{1 0} \% \text { vs. Referral } \\
\text { for A1c }<\mathbf{8} \%\end{array}$ \\
\hline Type of Surgery & 0.0783 & 0.647 & 1 & 0.522 & - & 0.920 \\
\hline Age & 1 & 1 & 1 & 1 & 1 & 1 \\
\hline Weight & 1 & 0.121 & 0.187 & 0.362 & 0.386 & 1 \\
\hline Height & 0.667 & 1 & 1 & 1 & 1 & 1 \\
\hline Body Mass Index & 1 & 0.00440 & 0.0142 & 0.100 & 0.116 & 0.791 \\
\hline A1c & $<0.00001$ & $<0.00001$ & $<0.00001$ & 1 & 1 & 1 \\
\hline Hematocrit & 1 & 1 & 1 & 1 & 1 & 1 \\
\hline Creatinine & 0.00121 & 1 & 0.317 & 0.0224 & 0.00038 & 0.592 \\
\hline Potassium & 0.594 & 1 & 1 & 1 & 1 & 1 \\
\hline Length of Stay & 0.332 & 1 & 1 & 1 & 1 & 1 \\
\hline Prolonged Length of Stay & $<0.001$ & 0.0404 & - & 0.362 & 0.296 & 0.920 \\
\hline Complication Rate & $<0.0001$ & 0.124 & 1 & 0.0826 & 0.152 & 1 \\
\hline Coronary Artery Disease & 0.120 & 0.00272 & - & 0.218 & - & 0.313 \\
\hline Hypertension & 0.196 & 0.203 & 0.764 & 1 & - & - \\
\hline Hypercholesterolemia & 0.048 & 0.00617 & 0.427 & 0.377 & 1 & 0.450 \\
\hline $\begin{array}{l}\text { Chronic Obstructive } \\
\text { Pulmonary Disease }\end{array}$ & - & - & - & - & - & - \\
\hline $\begin{array}{l}\text { Peripheral Vascular } \\
\text { Disease }\end{array}$ & - & - & - & - & - & - \\
\hline Tobacco Use & 0.863 & 0.823 & - & 0.863 & - & - \\
\hline Insulin & - & - & - & 1 & - & - \\
\hline Oral Hypoglycemic Drug & - & - & - & 0.00497 & 0.150 & 0.740 \\
\hline $\begin{array}{l}\text { Beta-Adrenoceptor } \\
\text { Antagonist }\end{array}$ & 0.310 & 0.0843 & 1 & 0.527 & 0.888 & 0.476 \\
\hline Calcium Channel Blocker & 0.181 & 0.920 & - & 0.413 & - & - \\
\hline Nitrate & - & - & - & - & - & - \\
\hline $\begin{array}{l}\text { ACE inhibitor or ATII } \\
\text { blocker }\end{array}$ & 0.00273 & 0.000512 & 0.262 & 0.454 & 0.764 & 0.343 \\
\hline
\end{tabular}

Table 3. Incidence Proportion of Postoperative Complications in Patients With and Without Diabetes Based on A1c Control Before elective Total Joint Surgery ${ }^{\text {a }}$

\begin{tabular}{lcccc}
\hline Variables & -DM & +DM No Referral & +DM Referral for A1c $<\mathbf{1 0} \%$ & +DM Referral for A1c $<\mathbf{8} \%$ \\
\hline Number & 100 & 55 & 32 & 16 \\
\hline Cardiac & 0.06 & 0.11 & 0.13 & 0.06 \\
\hline Pulmonary & 0.02 & 0.07 & 0.16 & 0.13 \\
\hline Neurologic & 0.01 & 0.07 & 0 & 0 \\
\hline Urinary Tract Infection & 0.02 & 0.07 & 0.06 & 0 \\
\hline Transfusion & 0.11 & 0.27 & 0.19 & 0.06 \\
\hline Pain & 0.04 & 0.27 & 0.19 & 0.06 \\
Surgical Site Infection & 0.05 & 0.15 & 0.19 & 0.06 \\
\hline Delayed Wound Healing & 0.04 & 0.18 & 0 & 0.19 \\
Mortality & 0.01 & 0.07 & 0.03 & 0 \\
\hline
\end{tabular}

\footnotetext{
a Abbreviations: + and - DM, with and without diabetes mellitus, respectively; A1c, glycosylated hemoglobin concentration.
} 


\section{Discussion}

The current results confirm the findings of several previous studies (1-5) indicating that is diabetes an important risk factor for postoperative complications. The results further demonstrate that more stringent long-term control of the disease directed by preoperative anesthesia clinic referral reduces the incidence of prolonged hospitalization and complication rate during the first year after surgery in patients undergoing elective total joint arthroplasty. Several previous large-scale clinical trials established a link between diabetes and major postoperative complications in orthopedic surgery patients. A study of 751,340 patients from the United States Nationwide Inpatient Sample undergoing primary or revision total knee or hip arthroplasty revealed that patients with diabetes were more likely to develop pneumonia, suffer cerebrovascular accidents, require autologous blood product transfusion, and have greater hospital costs than those without diabetes (1). Marchant et al. showed that the risk of stroke, urinary tract infection, ileus, postoperative hemorrhage, transfusion, prolonged hospitalization, and mortality was greater in patients with poorly controlled diabetes undergoing total joint arthroplasty compared with those whose diabetes was well controlled (4). Fewer infectious complications (particularly those involving the urinary tract), but not the risk of revision, deep venous thrombosis, pulmonary embolism, or prosthesis infection, were also observed in diabetic patients undergoing orthopedic surgery when HbA1c < compared with $\geq 7 \%(3,20,21)$. When complicated by end-organ damage (presumably resulting from poor long-term control), diabetes was shown to be a strong predictor (odds ratio of 1.94) of 90-day overall complication risk in a tenyear study of 138,399 patients undergoing total hip arthroplasty in California (5). More recently, Harris et al. retrospectively examined 6,088 diabetic patients treated in the Veterans Health Administration system and showed that there was linear relationship between HbA1c and complications after total joint arthroplasty (2). Preoperative HbA1c $\geq 7$ also increased the relative risk of complications and 90-day mortality after surgery. The current results demonstrating a significant correlation between the number of complications sustained per patient and long-term control of blood glucose concentration also verified the previously observed linear relationship between complication rate and HbA1c that was reported in veterans undergoing total joint arthroplasty (2). Other studies of diabetic patients undergoing cardiac $(6-8,22)$ and major noncardiac $(23-26)$ have reached similar conclusions about the importance of long-term control of blood glucose concentration on complication rate and outcome. Identification and optimization of co-existing diseases, including diabetes, are important goals in the anesthesia preoperative clinic setting $(27,28)$. To date, short-term control of blood glucose concentration, most often entailing avoidance of hypoglycemia and profound hyperglycemia, and management of the cardiovascular and renal sequelae of diabetes receive primary attention during preoperative assessment of patients with the disease (29), whereas attempts to gain tighter long-term control have generally not received emphasis. From this perspective, the objective of the current investigation was to examine whether preoperative clinic identification of veterans with poor long-term control of diabetes (as defined byHbA1c $\geq 10$ in 2009-10 and $\geq 8$ in 2011-12)and subsequent referral of these patients to primary care for improved control of their disease would reduce postoperative complications determined up to one year after elective total joint arthroplasty. Our results support this contention, as the simple decision to refer these patients with poorly controlled diabetes from the anesthesia preoperative clinic to primary care or endocrinology resulted in progressive reductions in complication rate and the number of patients requiring a prolonged hospital stay. Indeed, the rate of complications in diabetics when all patients with HbA1c $\geq 8$ were referred to primary care $(0.50 \pm 0.89)$ was statistically indistinguishable from that observed in their healthy peers without the disease $(0.36$ $\pm 0.63 ; \mathrm{P}=1$ ) when the current relatively small sample size was considered. Whether use of American Diabetes Association recommendations (HbA1c < 7\%) (15) for chronic glycemic control would further reduce complication rate remains to be determined. However, the current data suggest that moderate, less rigorous long-term control of blood glucose concentration substantially improves outcome in the setting of elective total joint surgery by reducing complication rate to values similar to those observed in patients without diabetes. The current results in diabetic veterans support the general observations of Kamal et al. indicating that evaluation of patients scheduled to undergo complex orthopedic surgery in an anesthesia preoperative clinic reduces mortality, unplanned intensive care unit admissions, intensive care and high dependency unit lengths of stay, and cost (14). The current results should be interpreted within the constraints of several potential limitations. Almost all of patients enrolled in the study were men (97\%) because of the Veterans Affairs population, and whether the current findings can also be extended to women is unknown. The sample size studied here was relatively small and was unable to distinguish differences in organ-specific complications between groups as a result. A larger study including women would be useful to determine such potential differences in end-organ complications and whether the observed complications are gender-specific. Nevertheless, it is clear, based on the observations of several largescale clinical studies, that diabetes is a risk factor for cardiac, neurological, infectious, and wound healing complications after orthopedic surgery $(1,2,4,5)$. Veterans with uncontrolled or poorly controlled diabetes were significantly more likely to have coronary artery disease 
and hypercholesterolemia than those in whom diabetic control was more rigorous. It is likely that the greater incidence of cardiovascular disease contributed to the higher complication rate in patients with poorly versus moderately controlled diabetes. The results also require qualification because patients with diabetes were required to have a HbA1c level recorded within six months before surgery. HbA1c most likely reflects chronic blood glucose control over a three-month interval (15), but many patients in our hospital often do not receive routine primary care to allow this frequency of HbAic sampling. As a result, a six-month HbA1c sampling interval was chosen to indicate chronic preoperative control of blood glucose concentration. Whether the current results in patients undergoing elective orthopedic surgery are applicable in other surgical patients is unknown, but diabetes has been identified as a major risk factor for perioperative morbidity and mortality in noncardiac and cardiac surgery (6-8) and it seems likely that improved long-term glycemic control may also exert beneficial effects on outcome in other groups of surgery patients when approached prospectively. Finally, fewer total joint operations were performed in diabetic veterans with tighter chronic control of blood glucose concentration. The current investigation did not consider the benefits of delaying total joint replacement to obtain more stringent glycemic control in diabetic patients versus the relative risk of continued, often quite severe, functional limitations occurring as a result of primary chronic joint pathology. In summary, the current results demonstrate that anesthesia preoperative clinic referral of diabetic veterans to primary care for more rigorous glycemic control before elective total joint arthroplasty reduces the incidence of prolonged hospitalization and complication rate during the first year after surgery. These data support the contention that anesthesia preoperative clinic evaluation and intervention may be directly linked to improved patient outcome.

\section{Authors' Contributions}

Peter J. Kallio helped design the study, conducted the study, analyzed the data, and wrote drafts of and revisions the manuscript. This author has seen the original data, reviewed the analysis of the data, approved the final manuscript, and is the author responsible for archiving the data files. Jenea Nolan conducted the study, analyzed the data, and wrote drafts and revisions of the manuscript. This author has seen the original data, reviewed the analysis of the data, and approved the final manuscript. Amy C. Olsen conducted the study, analyzed the data, and wrote drafts and revisions of the manuscript. This author has seen the original data, reviewed the analysis of the data, and approved the final manuscript. Susan Breakwell helped design the study, analyzed the data, and edited the original and revised manuscript. This author has seen the original data, reviewed the analysis of the data, and approved the final manuscript. Richard Topp helped design the study, analyzed the data, and edited the original and revised manuscript. This author has seen the original data, reviewed the analysis of the data, and approved the final manuscript. Paul S. Pagel analyzed the data and edited the original and revised manuscript. This author has seen the original data, reviewed the analysis of the data, and approved the final manuscript.

\section{Funding/Support}

This material is the result of work supported by a 201214 Jonas Scholar Award (Peter J Kallio), Marquette University, Milwaukee, Wisconsin, USA.

\section{References}

1. Bolognesi MP, Marchant MJ, Viens NA, Cook C, Pietrobon R, Vail TP. The impact of diabetes on perioperative patient outcomes after total hip and total knee arthroplasty in the United States. $J$ Arthroplasty. 2008;23(6 Suppl 1):92-8.

2. Harris AH, Bowe TR, Gupta S, Ellerbe LS, Giori NJ. Hemoglobin A1C as a marker for surgical risk in diabetic patients undergoing total joint arthroplasty. J Arthroplasty. 2013;28(8 Suppl):25-9.

3. Lamloum SM, Mobasher LA, Karar AH, Basiony L, Abdallah $\mathrm{TH}$, Al-Saleh AI, et al. Relationship between postoperative infectious complications and glycemic control for diabetic patients in an orthopedic hospital in Kuwait. Med Princ Pract. 2009;18(6):447-52.

4. Marchant MJ, Viens NA, Cook C, Vail TP, Bolognesi MP. The im pact of glycemic control and diabetes mellitus on perioperative outcomes after total joint arthroplasty. J Bone Joint Surg Am. 2009;91(7):1621-9.

5. Soohoo NF, Farng E, Lieberman JR, Chambers L, Zingmond DS Factors that predict short-term complication rates after total hip arthroplasty. Clin Orthop Relat Res. 2010;468(9):2363-71.

6. Dronge AS, Perkal MF, Kancir S, Concato J, Aslan M, Rosenthal RA. Long-term glycemic control and postoperative infectious complications. Arch Surg. 2006;141(4):375-80.

7. Halkos ME, Lattouf OM, Puskas JD, Kilgo P, Cooper WA, Morris CD, et al. Elevated preoperative hemoglobin A1c level is associated with reduced long-term survival after coronary artery bypass surgery. Ann Thorac Surg. 2008;86(5):1431-7.

8. Halkos ME, Puskas JD, Lattouf OM, Kilgo P, Kerendi F, Song HK, et al. Elevated preoperative hemoglobin A1c level is predictive of adverse events after coronary artery bypass surgery. J Thorac Cardiovasc Surg. 2008;136(3):631-40.

9. Koochemeshki V, Salmanzadeh HR, Sayyadi H, Amestejani M, Salehi Ardabili S. The effect of diabetes mellitus on short term mortality and morbidity after isolated coronary artery bypass grafting surgery. Int Cardiovasc Res J. 2013;7(2):41-5.

10. Correll DJ, Bader AM, Hull MW, Hsu C, Tsen LC, Hepner DL. Value of preoperative clinic visits in identifying issues with potential impact on operating room efficiency. Anesthesiology. 2006;105(6):1254-9.

11. Ferschl MB, Tung A, Sweitzer B, Huo D, Glick DB. Preoperative clinic visits reduce operating room cancellations and delays. Anesthesiology. 2005;103(4):855-9.

12. Schiff JH, Frankenhauser S, Pritsch M, Fornaschon SA, SnyderRamos SA, Heal C, et al. The Anesthesia Preoperative Evaluation Clinic (APEC): a prospective randomized controlled trial assessing impact on consultation time, direct costs, patient education and satisfaction with anesthesia care. Minerva Anestesiol. 2010;76(7):491-9.

13. Sweitzer BJ. Preoperative screening, evaluation, and optimization of the patient's medical status before outpatient surgery. Curr Opin Anaesthesiol. 2008;21(6):711-8.

14. Kamal T, Conway RM, Littlejohn I, Ricketts D. The role of a multidisciplinary pre-assessment clinic in reducing mortality 
after complex orthopaedic surgery. Ann R Coll Surg Engl. 2011; 93(2):149-51.

15. American Diabetes A. Standards of medical care in diabetes-2010. Diabetes Care. 2010;33 Suppl 1:S11-61.

16. Bhamidipati CM, LaPar DJ, Stukenborg GJ, Morrison CC, Kern JA, Kron IL, et al. Superiority of moderate control of hyperglycemia to tight control in patients undergoing coronary artery bypass grafting. J Thorac Cardiovasc Surg. 2011;141(2):543-51.

17. Lazar HL, McDonnell MM, Chipkin S, Fitzgerald C, Bliss C, Cabral $\mathrm{H}$. Effects of aggressive versus moderate glycemic control on clinical outcomes in diabetic coronary artery bypass graft patients. Ann Surg. 2011;254(3):458-63.

18. Coursin DB, Connery LE, Ketzler JT. Perioperative diabetic and hyperglycemic management issues. Crit Care Med. 2004;32(4 Suppl):S116-25.

19. Peterfreund RA, Lee SA. Endocrine surgery and intraoperative management of endocrine conditions. In: Longnecker DE, Brown DL, Newman MF, Zapol WM editors. Anesthesiology. 2th ed. New York: McGraw Hill Medical; 2012. pp. 1112-33.

20. Adams AL, Paxton EW, Wang JQ, Johnson ES, Bayliss EA, Ferrara A, et al. Surgical outcomes of total knee replacement according to diabetes status and glycemic control, 2001 to 2009. J Bone Joint $\operatorname{Surg}$ Am. 2013;95(6):481-7.

21. Iorio R, Williams KM, Marcantonio AJ, Specht LM, Tilzey JF, Healy WL. Diabetes mellitus, hemoglobin A1C, and the incidence of total joint arthroplasty infection. J Arthroplasty. 2012;27(5):726-9 e1.

22. Sato H, Carvalho G, Sato T, Lattermann R, Matsukawa T, Schricker T. The association of preoperative glycemic control, intraoperative insulin sensitivity, and outcomes after cardiac surgery. J Clin Endocrinol Metab. 2010;95(9):4338-44.

23. Ata A, Valerian BT, Lee EC, Bestle SL, Elmendorf SL, Stain SC. The effect of diabetes mellitus on surgical site infections after colorec- tal and noncolorectal general surgical operations. Am Surg. 2010;76(7):697-702.

24. Koutsoumbelis S, Hughes AP, Girardi FP, Cammisa FJ, Finerty EA, Nguyen JT, et al. Risk factors for postoperative infection following posterior lumbar instrumented arthrodesis. J Bone Joint Surg Am. 2011;93(17):1627-33.

25. Olsen MA, Nepple JJ, Riew KD, Lenke LG, Bridwell KH, Mayfield J, et al. Risk factors for surgical site infection following orthopaedic spinal operations. J Bone Joint Surg Am. 2008;90(1):62-9.

26. Sehgal R, Berg A, Figueroa R, Poritz LS, McKenna KJ, Stewart DB, et al. Risk factors for surgical site infections after colorectal resection in diabetic patients.J Am Coll Surg. 2011;212(1):29-34.

27. Committee on S, Practice P, Apfelbaum JL, Connis RT, Nickinovich DG, American Society of Anesthesiologists Task Force on Preanesthesia E, et al. Practice advisory for preanesthesia evaluation: an updated report by the American Society of Anesthesiologists Task Force on Preanesthesia Evaluation. Anesthesiology. 2012;116(3):522-38.

28. Sweitzer BJ, Pilla M. Overview of preoperative assessment and management. In: Longnecker DE, Brown DL, Newman MF, Zapol WE editors. Anesthesiology. 2th ed. New York: McGraw Hill Medical; 2012. pp. 52-76.

29. American College of Cardiology/American Heart Association Task Force on Practice G, American Society of E, American Society of Nuclear C, Heart Rhythm S, Society of Cardiovascular A, Society for Cardiovascular A, et al. ACC/AHA 2007 guidelines on perioperative cardiovascular evaluation and care for noncardiac surgery: executive summary: a report of the American College of Cardiology/American Heart Association Task Force on Practice Guidelines (Writing Committee to Revise the 2002 Guidelines on Perioperative Cardiovascular Evaluation for Noncardiac Surgery). Anesth Analg. 2008;106(3):685-712. 